\title{
Mécanismes moléculaires de l'action des agents immunosuppresseurs
}

Les immunosuppresseurs ciclosporine et FK506 se lient à des immunophilines et, sous la forme de ce complexe, à la calcineurine, une protéine phosphatase activée par la calmoduline en présence de $\mathrm{Ca}^{2+}$. L'inhibition de l'activité phosphatasique de la calcineurine induite par la fixation du complexe immunophiline-médicament pourrait interférer avec les modifications posttraductionnelles de composants cytoplasmiques de certains facteurs de transcription, probablement NF-AT et $\mathrm{NF}-\kappa \mathrm{B}$, dont la translocation dans le noyau, nécessaire à l'activation du gène de l'IL-2, ne pourrait plus se produire. Ainsi serait bloquée la première étape de l'activation des lymphocytes $T$ lorsque leur récepteur reconnaît un antigène spécifique présenté par une molécule du complexe majeur d'histocompatibilité.

Götz Baumann Jean-François Borel

ADRESSES

G. Baumann : docteur ès sciences, chef de laboratoire. J.-F. Borel: professeur d'immunopharmacologie, sous-directeur. Sandoz Pharma Ltd., Recherches précliniques-immunologie,

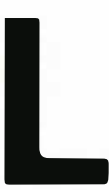

e peptide cyclique ciclosporine (CsA, Sandimmun ${ }^{(8)}$, isolé du champignon Tolypocladium inflatum gams, a été découvert il y a vingt ans, en janvier 1972 [1]. Depuis lors, ce peptide a révolutionné le domaine de la transplantation d'organes, et son efficacité est étudiée actuellement dans le traitement d'un certain nombre d'affections autoimmunes, en particulier le psoriasis, la polyarthrite rhumatoïde et le syndrome néphrotique [2]. Outre le succès de son introduction dans ces indications cliniques, la CsA a été aussi largement utilisée comme sonde expérimentale en recherche fondamentale, par exemple pour comprendre les voies de transduction des signaux et explorer des possibilités originales d'intervention pharmacologique [3]

\section{La ciclosporine se lie à la cyclophiline}

Des études immunologiques ont très tôt révélé que la CsA bloque l'acti- vation des cellules $T$ et que cela est en partie le résultat d'une inhibition de la transcription de gènes codant pour des lymphokines, notamment l'interleukine 2 (IL-2), facteur de croissance essentiel pour les cellules $\mathrm{T}$ [4]. Les concepts actuels concernant l'immunosuppression réalisée par la CsA suggèrent qu'en inhibant l'expression de l'IL-2 dans les cellules $\mathrm{T}$, la CsA empêche les cellules $\mathrm{T}$ auxiliaires d'orchestrer une réponse aux antigènes étrangers. Étant donné les effets spécifiques de la CsA sur la transcription des gènes de lymphokines dans les cellules $T$ et le rôle important que celles-ci jouent dans les rejets des greffes, les recherches concernant le mécanisme d'action de la CsA ont été essentiellement focalisées sur son rôle dans la régulation de l'expression des gènes dans les lymphocytes T. Au moins l'une des cibles intracellulaires de la CsA a été identifiée, et son activité enzymatique observée. Ce récepteur à haute affinité pour la CsA, appelé cyclophiline, appartient à une famille de plus en 


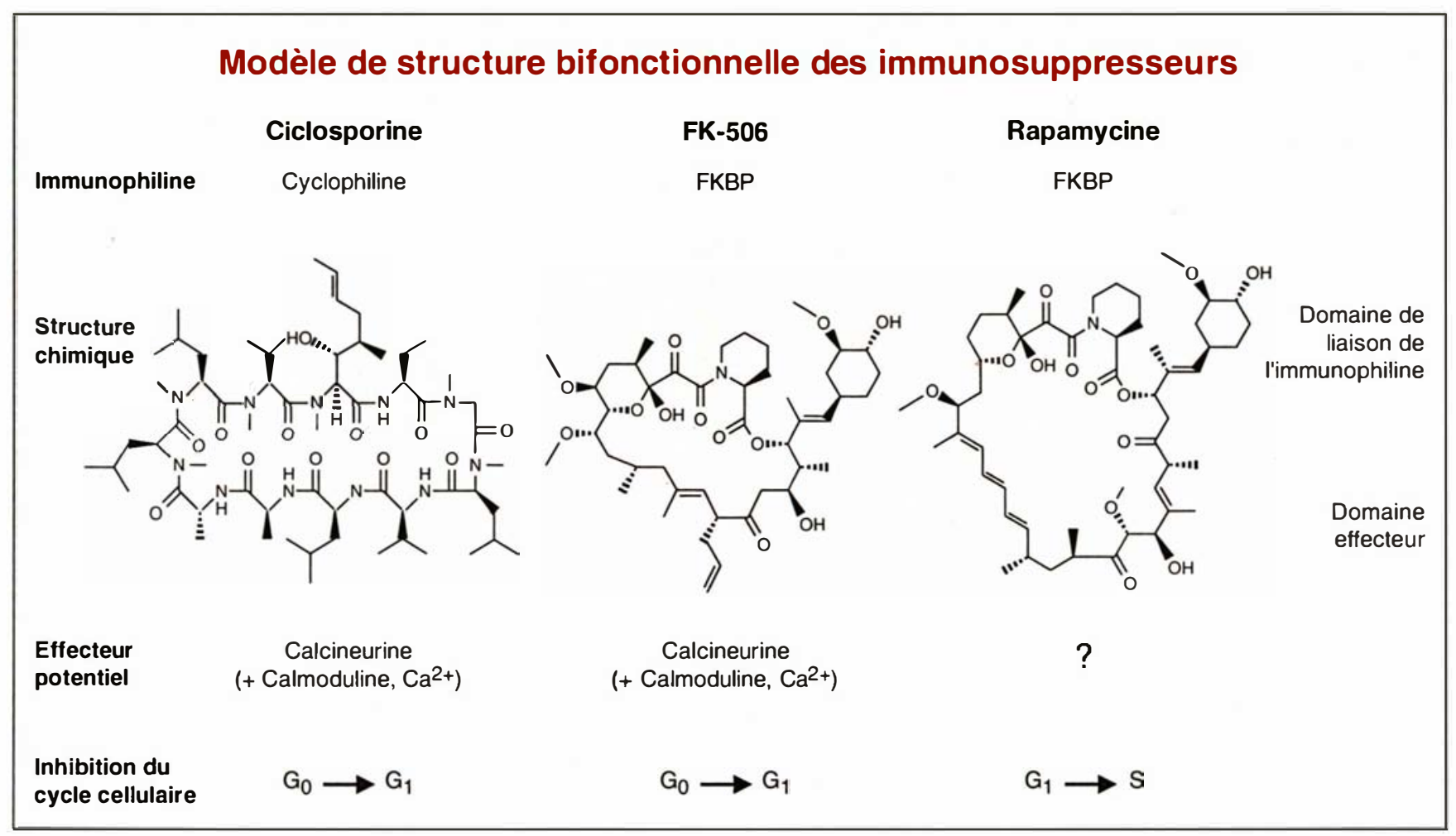

Figure 1. Conception du double système concernant les immunosuppresseurs CsA, FK-506 et rapamycine.

plus nombreuse d'immunophilines systémiques et spécifiques de tissus [5]. Tous les membres de cette famille identifiés jusqu'à présent possèdent une activité peptidyl-prolyl cistrans isomérase catalysant l'isomérisation cis-trans des liaisons peptidiques intéressant les résidus prolyl [6]. Cette activité enzymatique qui facilite le repliement des protéines est puissamment inhibée lorsque la protéine se lie à la CsA. Une première série d'arguments concernant les effets biologiques de la CsA sur la cyclophiline a été obtenue en comparant la capacité de liaison d'un grand nombre d'analogues de la CsA à la cyclophiline avec leurs activités immunosuppressives in vitro [7]. Tous les analogues de la CsA montrant une activité immunosuppressive significative se lient également à la cyclophiline. De plus, à la suite d'une approche génétique analysant chez les eucaryotes inférieurs (Neurospora crassa, Saccharomyces cerevisiae), les mutants de la cyclophiline qui ne se lient pas à la CsA, Tropschug et al. purent mon$\mathrm{m} / \mathrm{s} \mathrm{n}^{\circ} 4$, vol. 8 , auril 92

trer que l'effet cytotoxique de la CsA était relayé par la cyclophiline [8].

\section{Les macrolides FK-506 et rapamycine se lient également à une immunophiline (FKBP)}

On a découvert plus récemment un produit de type antibiotique, sans relation avec la CsA et provenant de Streptomyces tsukubaensis, doté d'effets inhibiteurs sur l'activation des cellules $\mathrm{T}$ étonnamment analogues à ceux de la CsA [9]. Cette molécule, connue sous le sigle FK-506, est un macrolide qui se lie à un groupe distinct de protéines réceptrices appelées protéines de liaison du FK (FKBP) [10]. Ni la CsA ni le FK-506 ne montrent de réaction croisée avec les récepteurs (c'est-à-dire les immunophilines) de l'autre. Étant donné que les deux médicaments bloquent l'induction de la transcription de gènes de cytokines à un stade précoce de l'activation des cellules $T$ auxiliaires induite par l'antigène (transition $G_{0}$ à $G_{1}$ du cycle cellulaire), on admet que l'activation des cellules $T$ nécessite l'activité distincte des deux immunophilines. Cette analogie fonctionnelle a été soulignée par la constatation que les cyclophilines et les FKBP ont une activité peptidyl-prolyl cis-trans isomérase et que cette activité est bloquée par la liaison du produit correspondant $\left(\mathrm{m} / \mathrm{s} n^{\circ} 10, \mathrm{vol} .6\right.$, p. 1022). Ces données d'observation suggérèrent tout d'abord que l'immunosuppression provenait d'un défaut de repliement du facteur de transcription nécessaire à l'expression de l'ARNm de la lymphokine. D'après ce modèle, on pensait que l'inhibition de l'isomérase de la proline était la base de l'inhibition des cellules $\mathrm{T}$ par la CsA et par le FK-506.

Les conceptions se sont ensuite radicalement modifiées à la lumière des résultats obtenus avec un autre immunosuppresseur, le macrolide rapamycine isolé de Streptomyces hygroscopicus. Ce composé inhibe l'activa- 


\section{RÉFÉRENCES}

1. Borel JF, Kis ZI. The discovery and development of cyclosporine (Sandimmune). Transplant Proc 1991; 23 : 1867-74.

2. Borel JF, Di Padova F, Mason J, Ques niaux V, Ryffel B, Wenger R. Pharmacology of cyclosporine (Sandimmunc). Phannacol Rev 1989 ; 41 : 239-434.

3. Hohman RJ, Hultsch T. Cyclosporin A : new insights for cell biologists and bioche mists. New Biologist $1990 ; 2$ : 663-72.

4. Krönke M, Lconard WJ, Depper JM, e al. Cyclosporin $\mathrm{A}$ inhibits $\mathrm{T}$-cell growth fac tor genc-cxpression at the level of mRNA transcription. Proc Natl Acad Sci USA 1984 ; 81: 5214-8.

5. Handschumacher RE, Harding MW Ricc J, Drugge RJ, Speicher DW. Cyclophilin : a specific cytosolic binding protein for cyclosporin A. Science $1984 ; 226: 544-7$

6. Fischer G, Wittmann L,B, Lang K, Kief haber T, Schmid FX. Cyclophilin and peptidyl-prolyl cis-trans isomerase are probably identical proteins. Nature 1989 ; 337 : 476-8.

7. Quesniaux VFJ, Schreier MH, Wenger RM, Hicstand PC, Harding MW, Van Regenmortel MHV. Molecular characteris tics of cyclophilin-cyclosporinc interaction. Transplantation 1988 ; 46 : 23-8.

8. Tropschug M, Barthelmess IB, Neuper W. Sensitivity to cyclosporin A is mediated by cyclophilin in Neurospora crassa and Sac charomyces cerevisiae. Nature 1989 ; 342 : 953-5.

9. Kino $\mathrm{TH}$, Hatanaka $\mathrm{H}$, Miyata $\mathrm{S}$, et al. FK-506, a novel immunosuppressant isolated from a Streptomyces. II. Immunosuppressive effect of FK-506 in vitro. $J$ Antibio $1987 ; 40$ : 1256-65.

10. Sickierka JJ, Hung SHY, Poe M, Lin CS, Sigal NH. A cytosolic binding protein for the immunosuppressant FK-506 has peptidyl-prolyl isomerase activity but is dis tinct from cyclophilin. Nature 1989 ; 341 : 755-7.

11. Dumont FJ, Staruch MJ, Koprak SL, Melino MR, Sigal NH. Distinct mechanisms of suppression of murine $\mathrm{T}$ cell activation by the related macrolides FK-506 and rapamycin. J Immunol 1990 ; 144 : 251-8.

12. Schreiber SL. Chemistry and biology of the immunophilins and their immunosuppressive ligands. Science $1991 ; 251$ : 283-7.

13. Dumont FJ, Mclino MR, Staruch MJ, Koprak SL, Fischer PA, Sigal NH. The immunosuppressive macrolides FK-506 and rapamycin act as reciprocal antagonists in murine T cells. I Immunol 1990; 144 1418-24. tion des cellules $\mathrm{T}$ à des concentrations comparables à celles du FK-506, de structure apparentée, mais avec un mécanisme qui diffère manifestement de celui du FK-506 et de la CsA [11]. La rapamycine n'inhibe pas la transcription des gènes d'activation précoce des cellules $T$, en particulier de l'IL-2, mais semble bien plutôt bloquer en aval les phénomènes aboutissant à la prolifération des cellules $\mathrm{T}$, c'est-à-dire la voie de transduction du signal émanant de récepteurs des lymphokines, tels que le récepteur de l'IL-2 (transition des phases $G_{1}$ à $G_{s}$ ).

La rapamycine se lie également à la FKBP et inhibe l'activité peptidylprolyl cis-trans isomérase, sans inhiber la transcription de l'IL-2. Cela démontre clairement que l'inhibition de l'activité de la peptidyl-prolyl cistrans isomérase par la FKBP est en soi insuffisante pour être le médiateur de l'effet biologique du FK-506 [12]. On a montré en outre, par des études de compétition, que FK-506 et rapamycine doivent se lier à un récepteur intracellulaire commun : la rapamycine peut, en effet, agir comme un puissant antagoniste du FK-506 [13].

\section{Le concept \\ d'un double système régulateur des immunosuppresseurs}

Le modèle actuel permet de penser que les deux médicaments agissent par l'intermédiaire de deux systèmes régulateurs : un système de liaison à l'immunophiline FKBP, partagé avec le FK-506 et la rapamycine, et un système d'effecteur spécifique de l'un et l'autre produits [12].

De façon frappante, le même concept d'un double système a été mis en évidence pour la CsA. Le site de liaison sur la cyclophiline a été précisé par des méthodes immunochimiques [7], confirmées ultérieurement par analyse en résonance magnétique nucléaire [14] et par cristallographie aux rayons X [15]. La définition du site effecteur sur la molécule de ciclosporine a été réalisée par une sélection soigneuse d'un petit groupe de dérivés de la CsA, diffusant dans les cellules, se liant à la cyclophiline et inhibiteurs de la cis-trans isomérase, qui se sont cependant avérés non immunosuppresseurs (G. Zenke et al., travail en cours). On a montré que ce petit groupe d'analogues de la CsA annule l'effet immunosuppresseur de la CsA lorsqu'ils sont ajoutés à des cellules $\mathrm{T}$ en concentrations molaires supérieures à celles de la CsA. Leur effet inhibiteur sur l'activité cis-trans isomérase de la cyclophiline fournit des arguments convaincants témoignant, par analogie au FK-506 et à la FKBP, que l'inhibition de cette activité enzymatique soit n'a pas de signification, soit, du moins, est insuffisante pour expliquer l'immunosuppression réalisée par la CsA [16]. Cependant, la liaison de la CsA et du FK-506 à leurs immunophilines respectives est une condition préalable obligatoire à leur potentiel immunosuppresseur. Le site effecteur constitué, dans le cas de la CsA, par les résidus modifiés dans le groupe non immunosuppresseur des dérivés de la CsA entrant en compétition pour la liaison au récepteur est donc tout à fait essentiel à l'activité immunosuppressive. Restait la question de savoir dans quelle mesure CsA et FK-506, lorsqu'ils sont liés à leurs récepteurs (immunophilines), exercent leur activité immunosuppressive en agissant par l'intermédiaire de leur site effecteur sur différentes cellules primaires ou sur différents sites d'une cible primaire commune. Cette cible avait toutes les chances, a priori, d'être un composant de la voie de transduction du signal qui aboutit finalement à la transcription du gène de l'IL-2.

\section{Le complexe immunophiline- médicament comme effecteur unique}

Sur la base de cet ensemble de données, les équipes de S. Schreiber [17] et de I. Weissman [18] ont testé la notion selon laquelle le complexe médicament-récepteur agit comme effecteur unique. Ils ont utilisé le complexe immunophiline-médicament comme matrice d'affinité pour isoler des protéines cytoplasmiques qui reconnaissent de manière spécifique le double complexe. De façon surprenante, les complexes entre CsA et cyclophiline, comme pour FK-506 et FKBP, se lient au même groupe de 


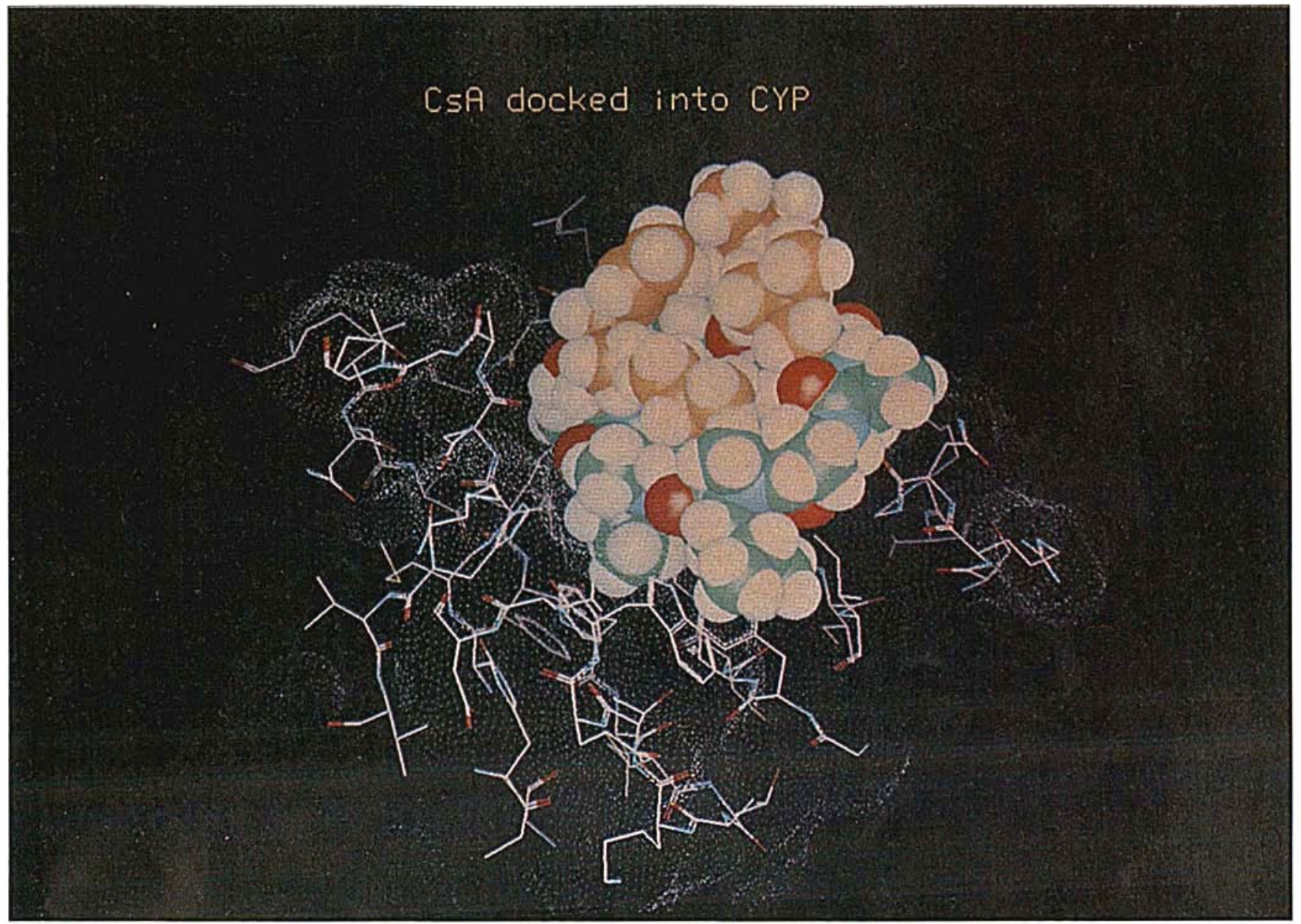

Figure 2. CsA (sphères pleines) insérée dans la structure cristalline de la cyclophiline humaine [15]. La conformation de la CsA a été déterminée par NMR [14] et son insertion dans le site actif de la cyclophiline effectuée par technique de modelage moléculaire (H.P. Weber, Sandoz Pharma S.A., Bâle).

protéines. Les deux groupes de travail ont pu montrer que les complexes immunophiline-médicament (mais non la cyclophiline, la FKBP ou la FKBP-rapamycine) se lient, de manière compétitive, directement à la calcineurine, une sérine-thréonine protéine phosphatase et, de manière indirecte, par l'intermédiaire de la calcineurine, à la calmoduline en présence de $\mathrm{Ca}^{2+}\left(\mathrm{m} / \mathrm{s} n^{\circ} 8\right.$, vol. 7, p. 878). Comme CsA et FK-506 témoignent d'une spécificité pour les voies d'activation qui induisent une augmentation des concentrations intracellulaires de $\mathrm{Ca}^{2}+$ telles que celles commandées par le récepteur de la cellule $T$, la calcineurine peut éventuellement être impliquée dans la régulation de l'état de phosphorylation des composés en aval de cette voie. De fait, de la calcineurine sti$\mathrm{m} / \mathrm{s} n^{\circ} 4$, vol. 8 , avril 92 mulée par la calmoduline, en présence de $\mathrm{Ca}^{2+}$, est puissamment inhibée in vitro par les complexes CsA-cyclophiline et FK-506 - FKBP. Des études biologiques ne doivent pas tarder à déterminer si la calcineurine est la cible authentique de la CsA et du FK-506 in vivo et représente ainsi une molécule clef dans la voie de transduction du signal émanant du récepteur de la cellule T. Quoique les effets de la CsA et du FK-506 aient une spécificité tissulaire, leurs protéines de liaison - tels la calcineurine, la cyclophiline et le FKBP - sont très répandues. Cela pourrait être expliqué par leurs interactions sélectives avec des isoformes spécifiques de la calcineurine, des membres de la famille des immunophilines spécifiques des tissus, ou par l'existence de substrats cellulaires spécifiques de la calcincurine. Ces substrats sont les composants les plus vraisemblables de la voie de transduction du signal qui, finalement, aboutit à la transcription du gène de l'IL-2. Des candidats potentiels cssentiels pour l'activation de l'IL-2 sont les facteurs de transcription inductibles par l'antigène, le NF-AT (nuclear factor of activated $T$ cells) [19] et le NF- $\alpha$ B (facteur nucléaire se fixant au site $\beta$ du enhancer du gène codant pour les chaînes légères $\kappa$ des immunoglobulines [20]), décrits tous deux comme modifiés dans leur activité de liaison au promoteur de l'IL-2 par la CsA et le FK-506, mais non par la rapamycine $[21,22]$. Il est très vraisemblable que la liaison à l'ADN des deux facteurs de transcription dépende d'une modification posttraductionnelle entraînant une trans- 


\section{RÉFÉRENCES}

14. Weber G, von Freiberg B, Traber R, Braun $W$, Widmer $H$, Wüthrich $K$. The NMR-structure of cyclosporin A bound to cyclophilin in aqueous solution. Biochemistry 1991 ; 30 : 6563-74.

15. Kallen J, Spitzfaden C, Zurini MGM, $e t$ al. Structure of human cyclophilin and its binding site for cyclosporin A determined by $\mathrm{X}$-ray crystallography and NMR spectroscopy. Nature 1991; 353 : 276-9.

16. Sigal NH, Dumont F, Durette P, et al. Is cyclophilin involved in the immunosuppressive and nephrotoxic mechanism of action of cyclosporin A. J Exp Med 1991; $173: 619-28$

17. Liu J, Farmer JD, Lane WS, Fricdman J, Weissman I, Schreiber SL. Calcineurin is a common target of cyclophilincyclosporin A and FKBP - FK-506 complexes. Cell 1991 ; 66 : 807-15.

18. Fricdman J, Weissman I. Two cytoplasmic candidates for immunophilin action arc revealed by affinity for a new cyclophilin : one in the presence and one in the absence of CsA. Cell 1991; 66 : 799-806.

19. Emmel EA, Verweij CL, Durand DB, Higgins KM, Lacy E, Crabtrce GR. Cyclosporin A specifically inhibits function of nuclear proteins involved in $\mathrm{T}$ cell activation. Science $1989 ; 246$ : 1617-20.

20. Lenardo MJ, Baltimorc D. NF- $\mathrm{B}$ : a pleiotropic mediator of inducible and tissuespecific gene control. Cell $1989 ; 58$ : 227-9.

21. Mattila PS, Ullman KS, Fiering S. The actions of cyclosporin A and FK-506 suggest a novel step in the activation of $T$ lymphocytes. EMBO J 1990 ; 9 : 4425-33.

22. Baumann G, Geisse S, Sullivan $M$ Cyclosporin A and FK-506 both affect DNA binding of regulatory nuclear proteins to the human interlcukin-2 promoter. New Biologist $1991 ; 3$ : 270-8.

23. Ghosh S, Baltimore D. Activation in vitro of $\mathrm{NF}-\kappa \mathrm{B}$ by phosphorylation of its inhibitor IкB. Nature 1990 ; 344: 678-82.

24. Flanagan WM, Corthésy B, Bram RJ Crabtrec GR. Nuclear association of a $\mathrm{T}$ cell transcription factor blocked by FK-506 and cyclosporin A. Nature 1991; 352 : 803-7.

25. Klausner RD, Samelson LE. T cell antigen receptor activation pathways : the tyrosine kinase connection. Cell 1991; 64: 875-8.

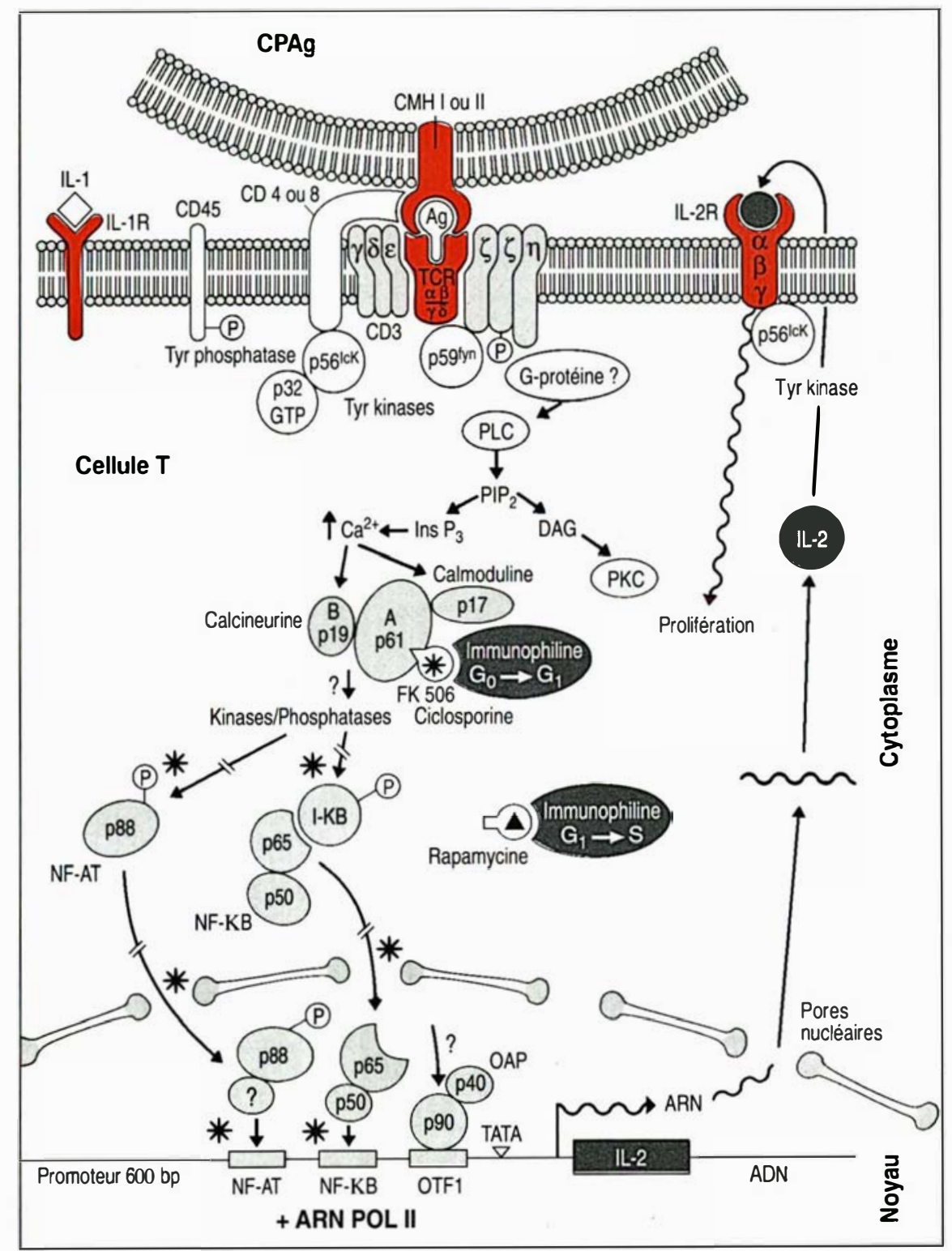

Figure 3. La CsA et le FK-506 interfèrent tous deux, par liaison à leur immunophiline respective, avec la fonction de molécules intracellulaires qui transmettent des signaux dépendant du $\mathrm{Ca}^{2+}$ entre le récepteur de la cellule T (TCR) et I'activation des gènes de I'interleukine 2 (IL-2) dans le noyau (revue générale, voir [25]). La régulation de la transcription du gène de I'IL-2 est modulée par l'association de facteurs de transcription (par exemple NF-AT, NF-kB, OTF-1) interagissant avec leurs sites spécifiques de reconnaissance sur le promoteur du gène de l'IL-2. Ces complexes ADN-protéines, avec I'ARN polymérase II (ARN pol II), aboutissent à la transcription induite par l'antigène du gène de I'IL-2. Les sites potentiels d'intervention du complexe pentamérique (calcineurine (p19/p61), calmoduline, immunophiline, médicament) mettant en jeu, par exemple, une modification post-traductionnelle des facteurs de transcription interférant avec leur translocation dans le noyau, sont indiqués (*). La CsA et le FK-506 interfèrent avec la transition $G_{0}$ à $G_{1}$ du cycle cellulaire alors que la rapamycine interfère avec la transition $G_{1}$ à $S$ (indiquée par un triangle). CPAg : cellule présentatrice de l'antigène; Ag : antigène; $\epsilon, \delta, \gamma, \eta, \zeta$ : sous-unités du complexe CD3. 
location nucléaire des précurseurs cytoplasmiques, translocation indispensable à la formation d'un complexe fonctionnel de transcription. Dans les cellules non stimulées, le $\mathrm{NF}-\kappa \mathrm{B}$ est lié sous une forme inactive à son inhibiteur I- $k \mathrm{~B}$. Lors de l'activation de la cellule $T, I-\kappa B$ est phosphorylé et libère, en conséquence, le NF- $\kappa$ B cytoplasmique, qui peut alors être transloqué dans le noyau [23]. Crabtree et al. ont démontré récemment que le NF-AT est formé lorsqu'un signal émanant du récepteur de l'antigène induit une sousunité cytoplasmique préexistante à se transloquer dans le noyau et à se combiner à une sous-unité nucléaire nouvellement synthétisée de NF-AT. CsA et FK-506, très vraisemblablement, bloquent la translocation en modifiant le composant cytoplasmique sans affecter la synthèse de la sousunité nucléaire [24].

\section{Conclusions}

En résumé, des données récentes concernant les mécanismes moléculaires de l'action de certaines médications immunosuppressives apportent des arguments solides en faveur du modèle fascinant selon lequel la CsA et le FK-506 agissent en se liant à des immunophilines et ensuite en inhibant, en tant que complexe médicament-immunophiline, la calcineurine, une protéine phosphatase activée par le calcium. Cette inhibition pourrait interférer avec des modifications post-traductionnelles des composants cytoplasmiques de facteurs de transcription, ce qui perturberait leur translocation nucléaire, condition préalable d'une transcription correcte du gène de l'IL-2. Tout se passe comme si les pièces de ce puzzle complexe de transduction de signaux commençaient à s'emboîter les uns avec les autres grâce à l'utilisation de métabolites microbiens immunosuppresseurs comme sondes moléculaires. Une fois connus les détails de la chaîne de processus qui ont lieu tout au long des voies de signalisation de la cellule $T$, les structures moléculaires en jeu fourniront de nouveaux outils à la recherche et un modèle rationnel pour la création d'agents thérapeutiques nouveaux, plus actifs et encore plus sélectifs $\mathrm{m} / \mathrm{s} n^{\circ} 4$, vol. 8 , auril 92

\section{Summary}

Molecular mechanism of immunosuppressive agents

Recent data on the molecular mechanism of some immunosuppressive drugs provide strong support for the fascinating postulate that CsA and FK-506 work by binding to immunophilins and then, as a drug-immunophilin complex, inhibiting the calciumactivated protein phosphatase, calcineurin. This inhibition could result in an altered modification pattern of the cytoplasmic components of transcription factors, thereby disturbing their nuclear translocation, which is a prerequisite for proper IL-2 transcription. It looks as if, with the immunosuppressive microbial metabolites as molecular probes, the pieces of this complex signal transduction puzzle are starting to fit together! Once the details of the chain of events along the $T$-cell signaling pathways are known, the molecular structures involved will provide new tools to be used in the search for and the rational design of new and improved therapeutic agents.
TIRÉS A PART

J.-F. Borel. 